\title{
Erratum to: Visualisation of the Zona Incerta for Deep Brain Stimulation at 3.0 Tesla
}

\author{
H. U. Kerl • L. Gerigk • S. Huck • M. Al-Zghloul • \\ C. Groden · I. S. Nölte
}

Published online: 8 May 2012

(C) Springer-Verlag 2012

Erratum to: Clin Neuroradiol (2012) 22:55-68

DOI: $10.1007 / \mathrm{s} 00062-012-0136-3$

The following text was missing:

H. U. Kerl and L. Gerigk contributed equally to this article

The original version of this article can be accessible under doi: 10.1007/s00062-012-0136-3

I. S. Nölte, MD $(\bowtie) \cdot$ H. U. Kerl, MD · S. Huck, MD ·

M. Al-Zghloul, MD · C. Groden, MD

Medical Faculty Mannheim,

Department of Neuroradiology, University of Heidelberg,

Theodor-Kutzer-Ufer 1-3, 68167 Mannheim, Germany

e-mail: ingo.noelte@umm.de

L. Gerigk, MD

Division of Radiology, German Cancer Research Centre,

Im Neuenheimer Feld 280, 69120 Heidelberg, Germany 